\title{
Review
}

\section{A brief review: acoustic emission method for tool wear monitoring during turning}

\author{
Xiaoli $\mathrm{Li}^{*}$ \\ School of Electric Engineering, Yanshuan University, Qinhuandao, 066004, P.R. China
}

Received 27 March 2001; received in revised form 28 June 2001; accepted 12 July 2001

\begin{abstract}
Research during the past several years has established the effectiveness of acoustic emission (AE)-based sensing methodologies for machine condition analysis and process monitoring. AE has been proposed and evaluated for a variety of sensing tasks as well as for use as a technique for quantitative studies of manufacturing processes. This paper reviews briefly the research on AE sensing of tool wear condition in turning. The main contents included are:

1. The AE generation in metal cutting processes, AE signal classification, and AE signal correction.

2. AE signal processing with various methodologies, including time series analysis, FFT, wavelet transform, etc.

3. Estimation of tool wear condition, including pattern classification, GMDH methodology, fuzzy classifier, neural network, and sensor and data fusion.
\end{abstract}

A review of AE-based tool wear monitoring in turning is an important step for improving and developing new tool wear monitoring methodology. (C) 2001 Elsevier Science Ltd. All rights reserved.

Keywords: Acoustic emission; Tool wear monitoring; Turning

\section{Introduction}

'Acoustic emission (AE) is the class of phenomena whereby transient elastic waves are generated by the rapid release of energy from a localized source or sources within a material, or the transient elastic wave(s) so generated' (ANSI/ASTM E 610-77). Clearly, an AE is a sound wave or, more properly, a stress wave that travels through a material as the result of some sudden release of strain energy. In recent years, AE instruments and systems have been developed for the monitoring and nondestructive testing of the structural integrity and general quality of a variety of materials, manufacturing processes, and some important devices.

Applications of $\mathrm{AE}$ for nondestructive testing are found in numerous industries, including refineries, pipe-

* Present address: Institute for Production and Machine Tools, Hannover University, Schlosswender Str. 5, 30159 Hannover, Germany. Fax: +49-511-762-5115.

E-mail address: lixiaoli_hit@yahoo.com (X. Li). lines, power generation (nuclear or other), aircraft, offshore oil platforms, paper mills and structures (bridges, cranes, etc.). AE products are also used for quality control in manufacturing operations and in research applications, and have important applications involving composite structures such as fiberglass, reinforced plastics and advanced aerospace materials.

Tool wear is a complex phenomenon occurring in different and varied ways in metal cutting processes. Generally, worn tools adversely affect the surface finish of the workpiece and therefore there is a need to develop tool wear condition monitoring systems which alert the operator to the state of tool, thereby avoiding undesirable consequences. Various methods for tool wear monitoring have been proposed in the past, even though none of these methods was universally successful due to the complex nature of the machining processes. These methods have been classified into direct (optical, radioactive and electrical resistance, etc.) and indirect (AE, spindle motor current, cutting force, vibration, etc.) sensing methods according to the sensors used. Recent 
attempts have concentrated on the development of the methods which monitor the cutting processes indirectly. Among these indirect methods, $\mathrm{AE}$ is one of the most effective for sensing tool wear. The major advantage of using $\mathrm{AE}$ to monitor tool condition is that the frequency range of the $\mathrm{AE}$ signal is much higher than that of the machine vibrations and environmental noises, and does not interfere with the cutting operation.

Grabec and Leskovar [1] first analyzed the sound emitted by the cutting process of aluminum alloy, and found that the spectrum of the audible frequency is discrete, while that of the ultrasonic range (later known as $\mathrm{AE}$ ) is continuous. They studied the influence of the relevant cutting parameters on the spectral distribution, and gave a qualitative explanation of the corresponding effects. The most important is to point out that AE could possibly be used to evaluate the sharpness of a cutting tool. Using an AE signal to monitor tool wear condition in cutting processes was started by Iwata and Moriwaki [2]. They found two important conclusions: the power spectrum of $\mathrm{AE}$ signals up to $350 \mathrm{kHz}$ increased with the tool wear and then reached saturation; and the total $\mathrm{AE}$ count was closely related to the tool wear. Since these initial reports, numerous studies have established the effectiveness of AE-based sensing methodologies for tool condition and cutting process monitoring.

In this paper, we review the AE-based tool wear condition monitoring in turning, which includes AE signal generation and correction in cutting processes, AE signal processing, and tool wear estimation.

\section{AE signal in the cutting process}

\subsection{AE signal source}

Research has shown that $\mathrm{AE}$, which refers to stress waves generated by the sudden release of energy in deforming materials, has been successfully used in laboratory tests to detect tool wear and fracture in singlepoint turning operations. Dornfeld (1989) [8] pointed out the following possible sources of $\mathrm{AE}$ during metalcutting processes (see Fig. 1):

(a) plastic deformation during the cutting process in the workpiece;

(b) plastic deformation in the chip;

(c) frictional contact between the tool flank face and the workpiece resulting in flank wear;

(d) frictional contact between the tool rank face and the chip resulting in crater wear;

(e) collisions between chip and tool;

(f) chip breakage;

(g) tool fracture.

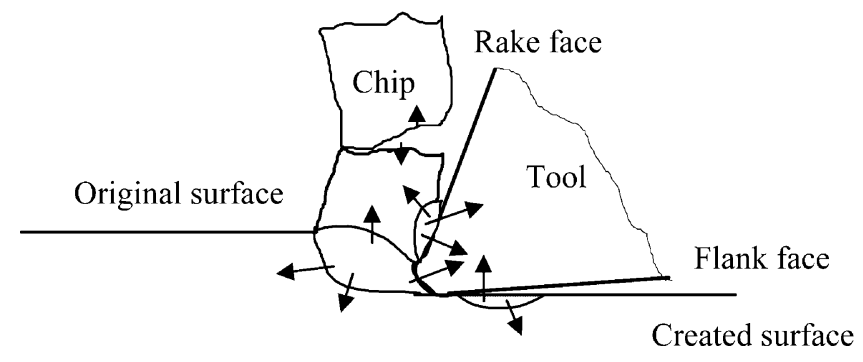

Workpiece

- Arrows indicate emitted AE

Fig. 1. AE generation during metal cutting.

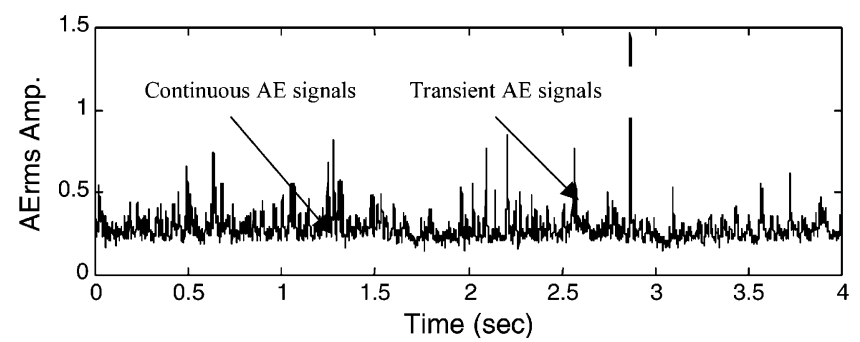

Fig. 2. Typical AE signals in turning. Cutting speed, $v=100 \mathrm{~m} / \mathrm{min}$; feed rate, $f=0.2 \mathrm{~mm} / \mathrm{rev}$; depth of cut, $d=2 \mathrm{~mm}$; workpiece material, middle steel, hardness $\mathrm{HB}=125$; cutting tools, CNMG 1204 04-QF.

\subsection{AE signal}

Based on the analysis of $\mathrm{AE}$ signal sources, $\mathrm{AE}$ derived from metal turning consists of continuous and transient signals, which have distinctly different characteristics. Continuous signals are associated with shearing in the primary zone and wear on the tool face and flank, while burst or transient signals result from either tool fracture or chip breakage. Therefore, from (a) to (d) sources generate continuous AE signals, while from (e) to $(\mathrm{g})$ generate transient $\mathrm{AE}$ signals (see Fig. 2). The AE signal types in cutting process show in Fig. 3.

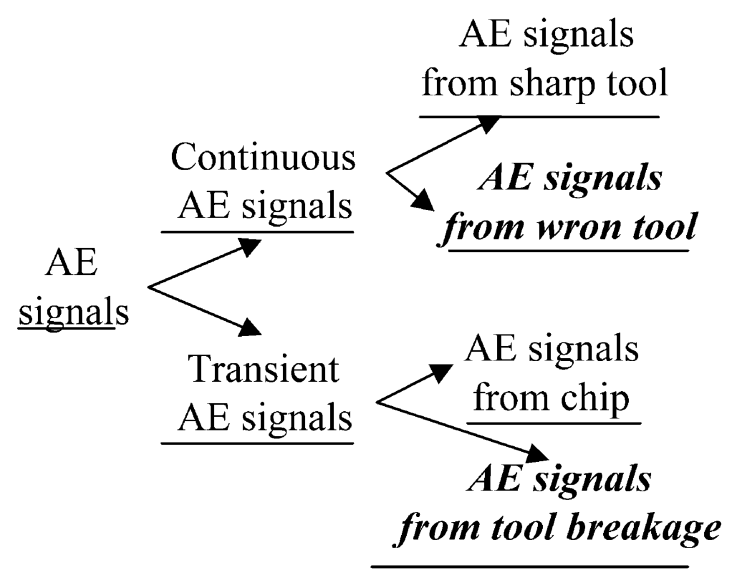

Fig. 3. AE signal type in cutting process. 
Uehara [3] has found that the amplitude of an AE signal from the workpiece is reduced decisively during $\mathrm{AE}$ transfer from workpiece to tool possibly by reflection at the interface. As a result, the friction between workpiece and tool and the tool fracture can be regarded as the most important sources of continuous and transient $\mathrm{AE}$ signals during turning, respectively. Therefore, the amplitude of the continuous-type AE signal could be used to monitor the wear of a cutting tool, as in [4,5].

In [5], continuous and discontinuous $\mathrm{AE}$ in turning are used to test gradual wear and intermittent degradation of cutting tools, respectively. The effects of machining parameters on characteristic AE obtained during machining were studied based on the frequency analysis of $\mathrm{AE}$ signal. In [6], the characteristic features of acoustic emission signals were analyzed during turning of medium carbon steel with a coated tool and uncoated tools. It was found that the AE signal changed from the burst type to a continuous type as the wear of the coated tool progressed and the ceramic coating was removed.

\subsection{AE signal correction}

Before studying AE signal in turning, we need to obtain an AE signal from the cutting system. The piezoelectric $\mathrm{AE}$ sensor was attached to the side of a tool shank. The AE signal was initially $40 \mathrm{~dB}$ pre-amplified and bandpass filtered. A bandpass filter with a range of $100 \mathrm{kHz}-1 \mathrm{MHz}$ was used to reduce the influence of low-frequency noise, and then the signal became fullwave rectified and averaged with a time constant of 1.2 $\mathrm{ms}$. The AE RMS voltage output was sampled by a computer at $2.5 \mathrm{kHz}$ (see Fig. 4).

\section{AE signal processing}

An AE signal is non-stationary and often comprises overlapping transients, whose waveforms and arrival times are unknown. A common problem in AE signal processing is to extract physical parameters of interest, such as tool wear, when these involve variations in both time and frequency. Many quantifiable characteristics of $\mathrm{AE}$ can be displayed as follows [7]:

Ring down count: the number of times the signal amplitude exceeds the present reference threshold;

$A E$ event: a micro-structural displacement that produces elastic waves in a material under load or stress; Rise time: the time taken to reach peak amplitude from the first present threshold voltage crossing of the signal;

Peak amplitude: this can be related to the intensity of the source in the material producing an AE signal; $R M S$ voltage: a measure of signal intensity.

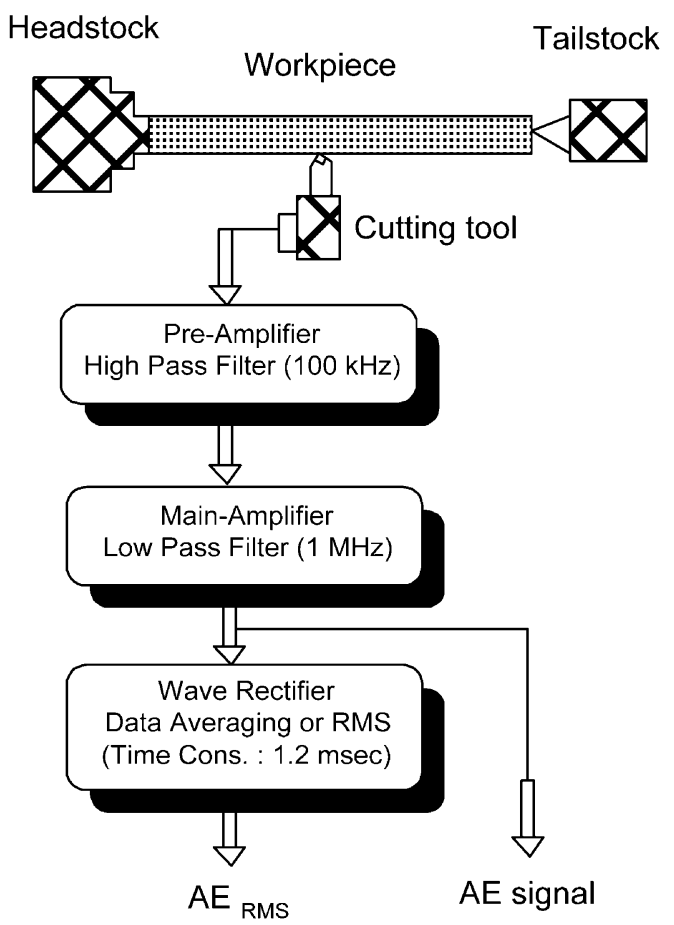

Fig. 4. AE signal correction and pre-processing.

Many signal processing methods have been used to analysis AE signals, with the aim to extract the features of AE signals for testing or monitoring. The main methods include time series analysis, fast Fourier transform (FFT), Gabor transform (or window (local) Fourier transform), Wigner-Ville distribution, and wavelet transform.

\subsection{Time series analysis}

In $[7,8]$, a time series modeling technique was used to extract parameters from $\mathrm{AE}$ signals acquired during turning. Autoregressive (AR) parameters and AR residual signals are taken as features for monitoring tool wear condition.

Set an AR model as follows:

$$
\begin{gathered}
\bar{x}(n)=a_{1} x(n-1)+a_{2} x(n-2)+\ldots+a_{5} x(n-5) \\
R(n)=\bar{x}(n)-x(n)
\end{gathered}
$$

where $x(n)$ is the RMS AE time series, $\bar{x}(n)$ is the AR predicted value, $a_{1}, a_{2}, \ldots, a_{5}$ are the AR model parameters, and $R(n)$ is the AR residual signal.

The experimental results have found that the power of the AR residual signal of the AE increases with increases of the flank wear of the cutter in turning. The AR parameters $a_{1}, a_{2}, \ldots, a_{5}$, were divided by $a_{5}$ to reduce the feature size to $A_{1}, A_{2}, A_{3}$, and $A_{4}$, and these parameters and the cutting parameters (cutting speed, $v \mathrm{~m} / \mathrm{min}$; feed rate, $f \mathrm{~mm} / \mathrm{rev}$; depth of cut, $d \mathrm{~mm}$ ) were taken as the input vectors for estimating tool wear condition.

Chung et al. [9] presented the application of $\mathrm{AE}$ and 
tool acceleration measurements to in-process assessment of machining quality in the context of tool wear and chatter. Adaptive time series modeling and band-filtered energy analysis were implemented in conjunction with the cutting condition classification based on artificial neural networks.

\subsection{Fourier transform}

An energy-limited signal $f(t)$ can be decomposed by its Fourier transform $F(w)$, namely

$f(t)=\frac{1}{2 \pi} \int_{-\infty}^{+\infty} F(w) \mathrm{e}^{\mathrm{i} w t} \mathrm{~d} t$

where

$F(w)=\int_{-\infty}^{+\infty} f(t) \mathrm{e}^{-\mathrm{i} w t} \mathrm{~d} t$

$f(t)$ and $F(w)$ are known as a pair of Fourier transforms. Eq. (2) implies that $f(t)$ signal can be decomposed into a family with harmonics $\mathrm{e}^{\mathrm{i} w t}$ and the weighting coefficient $F(w)$ represent the amplitudes of the harmonics in $f(t) . F(w)$ is independent of time, it represents the frequency composition of a random process, which is assumed to be stationary so that its statistics do not change with time.

Fourier transform has been successfully used to process the AE signal during turning. In [10], experimental results have shown that the magnitude of the $\mathrm{AE}$ in the frequency domain was sensitive to the change of tool state. However, the AE signal is essentially non-stationary. If we calculate the frequency composition of nonstationary signals by using Fourier transform, the results are the frequency composition averaged over the duration of the signal. As a result, Fourier transform cannot describe adequately the characteristics of the transient signal in the lower frequency.

\subsection{Gabor transform}

The Gabor transform or short-time Fourier transform (STFT) is the best known time-frequency technique, and it has been applied to signal processing, such as the complex AE signals [11]. In general, the STFT method is used to deal with non-stationary signals. STFT has a short data window centered at time. Spectral coefficients are calculated for this short length of data, the window is then moved to a new position and the calculation repeated. Assuming an energy-limited signal, $f(t)$ can be decomposed by STFT, namely

$$
G(w, \tau)=\int_{R} f(t) g(t-\tau) \mathrm{e}^{-\mathrm{i} w t} \mathrm{~d} t
$$

where $g(t-\tau)$ is called the window function. If the length of the window is represented by time duration $T$, its frequency bandwidth is approximately $1 / T$. Using a short data window means that the bandwidth of each spectral coefficient is of the order $1 / T$ and is therefore wide. A feature of the STFT is that all spectral estimates have the same bandwidth.

In [12], time-frequency representation of output spectrums of an AE sensor obtained from head-disk experiments giving evidence of stationary and non-stationary behavior is investigated. Gabor transform provides a natural framework for processing AE signals consisting of transients whose waveforms agree with the window function.

However, Gabor transform is badly adapted to signals where patterns with different scales appear, and it resolves short time phenomena associated with high frequencies poorly. Moreover, the representation coefficients can never be interpreted as local energy measures [13].

\subsection{Wavelet transform}

The basic idea behind signal processing with wavelets is that the signal can be decomposed into its component elements through the use of basic functions. In the case of wavelet analysis, the basic functions consist of the wavelet scale function, and scaled and shifted versions of the mother wavelet. Unlike in Fourier analysis, the basic function is sine or cosine waves.

Given a time-varying signal $f(t)$, the wavelet transform (WT) consisted of computing a coefficient that is the inner product of the signal and a family of wavelets. The wavelet transform can be given by

$$
\begin{aligned}
& \psi_{j, k}(t)=2^{-\frac{i}{2}} \psi\left(2^{-j} t-k\right) \\
& d_{0,0}=\langle f(t), \phi(t)\rangle \\
& d_{j, k}=\left\langle f(t), \psi_{j, k}(t)\right\rangle, j=1, \ldots, N, k=1, \ldots, 2^{j-1}
\end{aligned}
$$

where the $\psi_{j, k}(t)$ is the scaled and shifted version of the mother wavelet function $\psi(t), \phi(t)$ is the scale function, $d_{j, k}$ are the wavelet coefficients, and $N$ is the number of wavelet scales over which the wavelet transform is generated, usually chosen as a power of 2 .

Kamarthi et al. [14] dealt with the representational and analysis issues of AE signals in turning processes. The effectiveness of the wavelet representation of AE signals is studied in the context of flank wear estimation problem in turning processes. A set of turning experiments is conducted in which the flank wear is monitored through AE signals. Specially designed neural network architecture is used to relate AE features to flank wear. The accurate flank wear estimation results indicate that the wavelet transform representation of $\mathrm{AE}$ signals is very effective in extracting the $\mathrm{AE}$ features sensitive to gradually increasing flank wear. 
Li [15] presented a real-time tool breakage detection method for small-diameter drills using $\mathrm{AE}$ and current signals. The features of tool breakage were obtained from the $\mathrm{AE}$ signal using typical signal processing methods. The continuous wavelet transform (CWT) and the discrete wavelet transform (DWT) were used to decompose the spindle current signal and the feed current signal, respectively. The tool breakage features were extracted from the decomposed AE signals. In [16], a wavelet transform can decompose AE signals into different frequency bands in the time domain. The root mean square (RMS) values extracted from the decomposed signal for each frequency band was used as the monitoring feature for tool wear. Chen et al. [17] demonstrated the sensitivity of acoustic emission to tool-work contact and chip thickness in ultraprecision machining. A signal processing scheme utilizing a wavelet transform that identifies the possible dominant cutting mechanism for a certain cutting state, given the $\mathrm{AE}$ signals from that process, was developed.

\section{Tool wear estimation}

The relationship between the AE signal and tool wear is not simple. Kim et al. [18] observed the purely progressive tool wear in turning operations. As a result, they found that in most experimental results the refined mean level (RML) of the averaged AE signal increases at first with an increase of flank wear, and then stays at an approximately constant level even with further increase of flank wear while the fluctuation of the RML across the constant level becomes rather high. Clearly, the relationship between the $\mathrm{AE}$ signal and tool wear condition is nonlinear, so the general mathematical relation cannot be used to map this relation.

If we can look for an effective mathematical model to map the relationship between the AE signal (some features) and tool wear, the AE signal could be used to monitor tool wear condition in real time for turning. Some models have been presented, such as (in [19]) a linear regression model developed to relate the flank wear of a carbide turning insert with the cumulative count of $\mathrm{AE}$, and the limiting value of the cumulative $\mathrm{AE}$ count for the limiting flank wear was predicted using this model. In [20], the AE count rate has been found to be a reliable parameter for predicting the flank wear of a cutting tool in real time. A linear regression model has been developed to relate the flank wear of a carbide turning insert with the cumulative count of AE.

In [21], the correlation between intrinsic frequencies and $\mathrm{AE}$ sources is identified by examining the RMS, dominant amplitude, type, and count rate of the AE signals. The tool life estimated from the RMS of the AE signal is shown to be in good agreement with that determined from measurements of the maximum wears and width on the tool nose. The results obtained demonstrate that $\mathrm{AE}$ is an effective technique for in-process wear monitoring and wear mechanism identification of multilayer ceramic-coated tools.

To effectively monitor different tool wear conditions, while avoiding the effect of other factors such as cutting parameters, some methodologies have been presented, as follows.

\subsection{Pattern classification methodology}

Pattern classification is a problem of separating each cluster pattern from a given source into a region characteristic of that particular class. For this, define a set of scalar functions, $\left\{f_{j}(X)\right\}$, where $X$ is the given pattern, and generated by source $C_{i}$, the function $f_{j}(X)$ will be maximized, i.e.

$\max _{1 \leq j \leq C}\left\{f_{j}(X)\right\}=f_{j}(X)$, if $X \in C_{i}$.

For computational simplicity, $\left\{f_{j}(X)\right\}$ are chosen to be linear functions, and a least mean squared error design is used to obtain the weights of these functions.

Emel and Kannatey-Asibu [22,23] applied a pattern classification methodology for sensing tool failure in turning. They developed a linear discriminate functionbased technique for detection of tool wear, tool fracture, or chip disturbance events by using the spectra of $\mathrm{AE}$ signals. In [24], the combined output of radial force, feed force and AE (RMS value) is utilized to model the tool flank wear in a turning operation. The tool wear sensing system consists of two phases: training and classification. The training phase is done off-line and is used to determine the weight coefficients for the linear decision functions using the prototype patterns from the cutting tests. The classification phase is in real time. The linear decision functions of the prototype test selected are used for classifying the incoming signal of the actual cutting test into one of three wear classes.

\subsection{GMDH methodology}

The group method of data handing (GMDH) [25] was developed in the 1960s by Ivaknenko. The algorithm is able to contract a regressive mathematical model of high order, which can accept a large number of variables and automatically organize a gradually more complex model until an optimal model is attained.

Suppose that the phenomenon under observation is regulated by $m$ independent variables $x_{1}, x_{2}, \ldots, x_{m}$, and we can record a large number $n$ of observations of variables $m$ and of the dependent variable $y$ for several cases of the phenomenon studied. The simplest equations that can be written are those that take all the variables in pairs as if only one pair is meaningful enough to describe the 
phenomenon. For example, observations with the generic pair of variables $x_{i}, x_{j}$, can be written;

$y=a+b x_{i}+c x_{j}+d x_{i} y_{j}+e x_{i}^{2}+f x_{j}^{2}$

In [26], the decision tree method and the GMDH were adopted for the recognition and prediction of the tool wear state in a turning operation using acoustic emission and cutting force signals. The GMDH algorithm determines a representation of the real-time machining system interrelationship between tool flank wear and the quantitative measure of sensor variables involved. The derived model was used to predict the tool wear from the inprocess sensor output features.

\subsection{Fuzzy classifier}

Fuzzy $c$-means algorithm is one of the most popular methods in the fuzzy classifier. In this approach, the aim in clustering is to determine the cluster centers, which are representative values of features corresponding to the classified categories. Once clustering centers are determined at the learning stage, then the classification is made by the comparison of the incoming pattern and each clustering center.

Let $X=\left\{X_{1}, X_{2}, \ldots, X_{n}\right\} \subset R$, where each $X_{i}=\left(x_{i 1}, x_{i 2}, \ldots, x_{i s}\right)$ $\in R$ is a feature vector; $x_{i j}$ is the $j$ th feature of individual $x_{i}$. For each integer $c, 2 \leq c<n$, let $V_{c n}$ be the vector space of $c \times n$ matrices with entries in [0,1], and let $u_{i j}$ denote the $i j$ th element of any $U \in V_{c n}$. The function $u_{i}: X \rightarrow[0,1]$ becomes a membership function and is called a fuzzy subset in $X$. Here $u_{i j}=u_{i}\left(x_{j}\right)$ is called the grade of membership of $x_{j}$ in the fuzzy set $u_{i}$. In the space of samples, we suppose that there are $n$ samples, which can be divided into $c$ classes. Consider the following subset of $V_{c n}$ :

$$
\begin{aligned}
M_{\mathrm{fc}} & =\left\{U \in V_{c n} \mid u_{i j} \in[0,1] \forall i, j ; \sum_{i=1}^{c} u_{i j}=1 \forall j ; \sum_{i=1}^{c} u_{i j}\right. \\
& >0 \forall i\}
\end{aligned}
$$

Each $U \in M_{\mathrm{fc}}$ is called a fuzzy $c$-partition of $X ; M_{\mathrm{fc}}$ is the fuzzy $c$-partition space associated with $X$. For any real number $m \in[1,5]$, define the real-valued functional $J: M_{\mathrm{fc}} \times L_{\mathrm{c}} \rightarrow R$ by

$J(U, V)=\sum_{k=1}^{n} \sum_{i=1}^{c}\left(u_{i k}\right)^{m}\left\|x_{k}-v_{i}\right\|^{2}$

$1 \leq m<\infty$, and usually $m=2$. where $U=\left\{u_{i k}\right\}$ is the membership function, with $u_{i k} \in[0,1]$, which denotes the degree of membership of the $k$ th pattern and $i$ th cluster centers; $V=\left\{v_{1}, v_{2}, \ldots, v_{c}\right\}$ is a vector of $c$ clusters. These $v_{i}$ are interpreted as clusters defined by their companion $U$ matrix, and play a fundamental role in our develop- ment. The functional $J$ is a weight, least squares objective function. In order to obtain the optimum fuzzy partition, this objective function must be minimized, i.e.

$\operatorname{minimize}\{J(U, V)\}$

The optimal solution to the above equation is that

$$
\begin{aligned}
& u_{i k}=\frac{1}{\sum_{j=1}^{c}\left(\frac{\left\|X_{k}-V_{i}\right\|}{\left\|X_{k}-V_{j}\right\|}\right)^{2 /(m-1)}}, \forall i, k \\
& V_{i}=\frac{\sum_{k=1}^{n}\left(u_{i k}\right)^{m} X_{k}}{\sum_{k=1}^{n}\left(u_{i k}\right)^{m}}, \forall i
\end{aligned}
$$

Suppose that under a given cutting condition, the features of training data sets determine a clustering center. Then all subsequent observations can be classified by using Eq. (11). That is

$u_{k 0}=\frac{1}{\sum_{j=1}^{c}\left(\frac{\left\|X_{0}-V_{i}\right\|}{\left\|X_{0}-V_{j}\right\|}\right)^{2 /(m-1)}}, \forall i, k$

where $u_{k 0}$ is the fuzzy grade of the current observation being assigned to the $k$ th wear state category and $X_{0}$ is the current observation.

In [10], a wavelet packet transform is used to capture the features of the AE signal, which are sensitive to the changes in tool wear condition, but are insensitive to the variation in process working conditions and various noises. The extracted features are classified by using the fuzzy ISODATA algorithm. As a result, the tool wear condition can be estimated over a wide range of cutting conditions for boring.

\subsection{Neural networks}

Neural networks are organized in layers each consisting of neurons or processing elements that are interconnected. There are a number of learning methods to train neural nets but the back-propagation (back-prop) paradigm has emerged as the most popular training mechanism. The back-prop method works by measuring the difference between output and the observed output value. The values being calculated at the output layer are propagated to the previous layers and used for adjusting the connection weights. Fig. 5 shows a typical multilayered feedforward neural network.

In [27], a class of polynomial learning network (PLN) models is used to identify cutting tool conditions; these multilayered networks have a self-organizing control structure based on the mechanisms of exhibition and 
Inputs

(The features of AE signal, cutting parameters, and others)

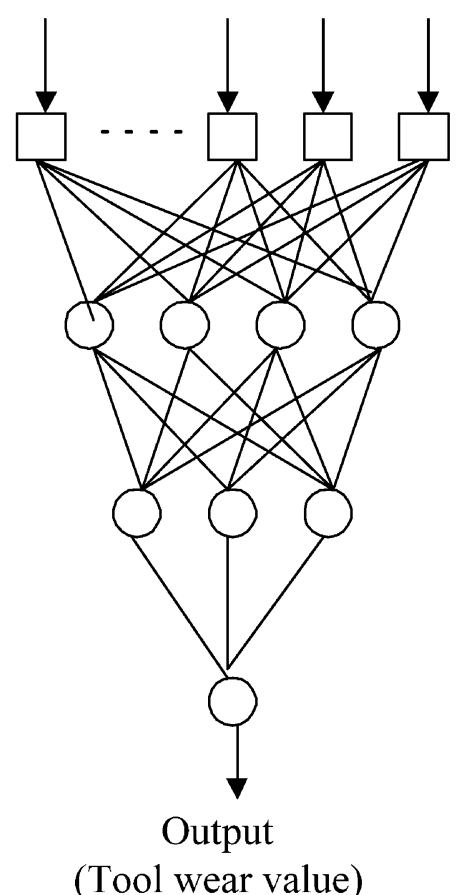

Fig. 5. Typical multilayered feedforward neural network.

inhibition. AE signals from an interrupted face-turning operation are modeled, and tool evolution from normal, cracked and finally broken tools are discriminated through network connectivity and node weights. However, feedforward neural networks require expensive training information and cannot remain adaptive after training. In $[28,29]$, an unsupervised ART2 neural network is used for the fusion of $\mathrm{AE}$ and force information and decision making of the tool flank wear state.

In order to overcome neural network drawbacks, a hybrid model of neural network and fuzzy logic (fuzzy neural network) is presented. There are many possible combinations of the two systems; the typical fuzzy neural network is shown in Fig. 6.

In [30], a fuzzy neural network is used to describe the relation between the monitoring features, which are

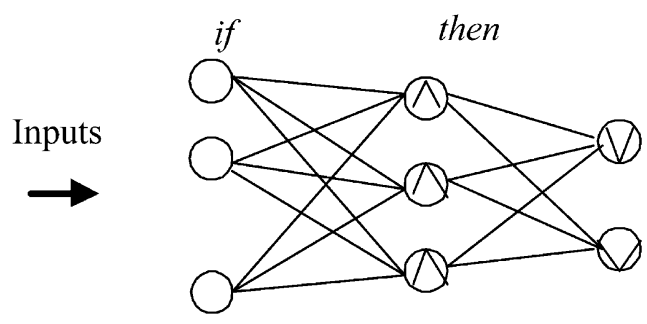

Outputs

Fig. 6. Typical fuzzy neural network. derived from wavelet-based $\mathrm{AE}$ signals, and the tool wear condition.

\subsection{Sensor and data fusion methodology}

The definition of data fusion derived from Subpanel is as follows: 'data fusion is a multilevel, multifaceted process dealing with the automatic detection, association, correlation, estimation, and combination of data and information from multiple sources.'

In tool wear monitoring systems, the architectures of sensor and data fusion include three types, see Fig. 7.

Based on the type (a), Rangwala and Dornfeld [31] presented a multiple sensor scheme utilizing cutting force and $\mathrm{AE}$ information, and applied a multilayer, feedforward neural network with error back-propagation to monitor tool wear states in a turning operation. In [32], adaptive time series modeling and band-filtered energy analysis are implemented in conjunction with the tool wear condition classification based on an artificial neural network. In [33], a novel parallel multi-ART2 neural network has been developed to estimate tool wear condition. Using the maximum frequency-band coherence function of two acceleration signals and the relative weighted frequency-band power ratio of an $\mathrm{AE}$ signal as input features, the neural network was used to identify various tool failure states in turning operations.

(a)

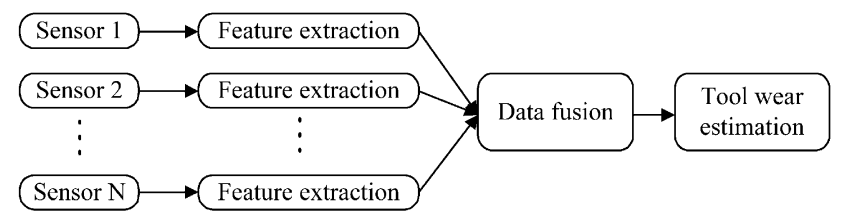

(b)

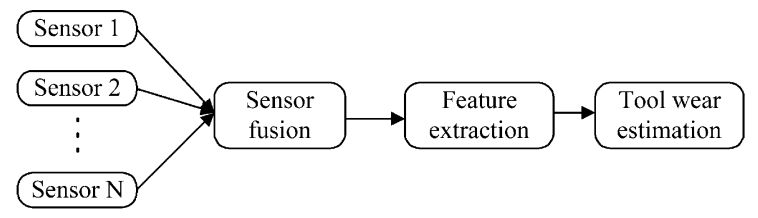

(c)

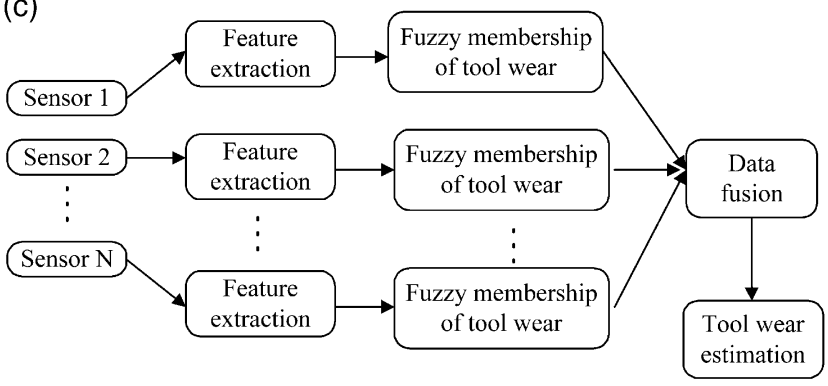

Fig. 7. Tool wear estimation using sensor and data fusion. 
In [34], AE sensor and cutting force sensor is combined in a new sensor based on type (b) of Fig. 7, which is able to measure $\mathrm{AE}$ and cutting force components simultaneously. The new sensor was used to reliably monitoring tool condition in turning.

In [35], $\mathrm{AE}$ and motor current signals are measured to identify tool wear condition using fuzzy classification, then the fuzzy values of tool wears with $\mathrm{AE}$ and motor current are fused by fuzzy logic (rule) to estimate tool wear in turning. Clearly, the monitoring system belongs to type (c) of Fig. 7.

\section{Conclusions}

Currently, AE-based sensing technology is the area of most intense research activity for developing intelligent tool condition systems. The reason is that the sensitivity of $\mathrm{AE}$ to tool wear and fracture is coupled with a high response rate of the signal. However, AE signals are heavily depended on process parameters. Thus, a key issue is how to reduce these effects in intelligent tool wear and fracture monitoring using AE signals. Based on this review, careful signal processing or feature extraction and integration with other sensor(s) will be an effective approach for AE-based tool condition monitoring.

\section{Acknowledgements}

This work described in this paper was fully supported by a grant from the City University of Hong Kong, Hong Kong (Project No. 7100063).

\section{References}

[1] P. Grbec, P. Leskovar, Acoustic emission of a cutting process, Ultrasonics 15 (1) (1977) 17-20.

[2] K. Iwata, T. Moriwaki, Application of acoustic emission measurement to in-process sensing of tool wear, Ann. CIRP 26 (1-2) (1977) 19-23.

[3] K. Uehara, Identification of chip formation mechanism through acoustic emission measurement, Ann. CIRP 33 (1) (1984) 71-74.

[4] K. Kojima, I. Inasaki, R. Miyake, Monitoring of turning process with acoustic emission signals, Nippon Kikai Gakkai Ronbunshu, C Hen 52 (474) (1986) 799-805.

[5] Y. Naerheim, A. Arora, In-process monitoring of machining using acoustic emission, in: Review of Progress in Quantitative Nondestructive Evaluation, 3 (B), Plenum Press, USA, 1984, pp. $753-762$.

[6] T. Moriwaki, M. Tobito, New approach to automatic detection of life of coated tool based on acoustic emission measurement, American Society of Mechanical Engineers, Production Engineering Division (Publication) PED 33 (1988) 75-82.

[7] H. Ravindra, Y. Srinivasa, R. Krishnamurthy, Acoustic emission for tool condition monitoring in metal cutting, Wear 212 (1) (1997) 78-84.
[8] S. Liang, D. Dornfeld, Tool wear detection using time series analysis of acoustic emission, J. Eng. Ind. Trans. ASME 111 (3) (1989) 199-205.

[9] E. Chung, Y. Chiou, S. Liang, Tool wear and chatter detection in turning via time-series modeling and frequency band averaging, Manuf. Sci. Eng. Am. Soc. Mech. Eng. PED 64 (1993) 351-358.

[10] X. Li, Z. Yuan, Tool wear monitoring with wavelet packet transform-fuzzy clustering method, Wear 219 (2) (1998) 145-154.

[11] A. Menon, Z. Boutaghou, Time-frequency analysis of tribological systems-Part I: implementation and interpretation, Tribol. Int. 31 (9) (1998) 501-510.

[12] E. Serrano, M. Fabio, Application of the wavelet transform to acoustic emission signals processing, IEEE Trans. Signal Process. 44 (5) (1996) 1270-1275.

[13] C. Heil, D. Walnut, Continuous and discrete wavelet transforms, SIAM Rev. 31 (4) (1989) 628-666.

[14] S. Kamarthi, S. Kumara, P. Cohen, Wavelet representation of acoustic emission in turning process, Intell. Eng. Syst. Artif. Neural Netw. 5 (1995) 861-866.

[15] X. Li, Real-time detection of the breakage of small diameter drills with wavelet transform, Int. J. Adv. Manuf. Technol. 14 (8) (1998) 539-543.

[16] X. Li, Y. Yao, Z. Yaun, On-line tool condition monitoring system with wavelet fuzzy neural network, J. Intell. Manuf. 8 (4) (1997) 271-276.

[17] X. Chen, J. Tang, D. Dornfeld, Monitoring and analysis of ultraprecision metal cutting with acoustic emission, in: Proceedings of the ASME Dynamic Systems and Control ASME, New York, NY, USA, 1996, pp. 387-393.

[18] K. Kim, K. Kim, H. Hwang, Progressive tool wear sensing in turning operations via acoustic emission signal processing, American Society of Mechanical Engineers, Production Engineering Division (Publication) PED 21 (1986) 297-307.

[19] A. Sampath, S. Vajpayee, On-line tool wear prediction using acoustic emission, in: Emerging Trends in Manufacturing: Proceedings of the 12th All India Machine Tool Design and Research Conference Tata McGraw-Hill, 1986, pp. 269-272.

[20] S. Vajpayee, A. Sampath, Acoustic emission as an indirect parameter for tool monitoring, in: Proceedings of Manufacturing International '88, 1, ASME, NY, USA, 1988, pp. 321-325.

[21] S. Cho, K. Komvopoulos, Correlation between acoustic emission and wear of multi-layer ceramic coated carbide tools, J. Manuf. Sci. Eng. Trans. ASME 119 (2) (1997) 238-246.

[22] E. Emel, E. Kannatey-Asibu, Tool failure monitoring in turning by pattern recognition analysis of AE signals, J. Manuf. Sci. Eng. Trans. ASME 110 (2) (1988) 137-145.

[23] E. Emel, E. Kannatey-Asibu, Acoustic emission and force sensor fusion for monitoring the cutting process, Int. J. Mech. Sci. 31 (11-12) (1989) 795-809.

[24] S. Damodarasamy, S. Raman, Inexpensive system for classifying tool wear states using pattern recognition, Wear 170 (2) (1993) $149-160$.

[25] S.T. Farlow, Self-organizing Methods in Modeling, Marcel Dekker, New York, 1984.

[26] C. Jiaa, D. Dornfeld, Self-organizing approach to the prediction and detection of tool wear, ISA Trans. 37 (4) (1998) 239-255.

[27] S. Darenfed, S. Wu, Polynomial learning networks for cutting tool diagnosis in machining operations, Trans. Can. Soc. Mech. Eng. 16 (2) (1992) 147-163.

[28] Y. Niu, Y. Wong, G. Hong, Intelligent sensor system approach for reliable tool flank wear recognition, Int. J. Adv. Manuf. Technol. 14 (2) (1998) 77-84.

[29] E. Emel, Tool wear detection by neural network based acoustic emission sensing, American Society of Mechanical Engineers, Dynamic Systems and Control Division (Publication) DSC 28 (1991) 79-85.

[30] Y.X. Yao, X. Li, Z.J. Yuan, Tool wear detection with fuzzy 
classification and wavelet fuzzy neural network, Int. J. Machine Tools \& Manufacture 39 (1999) 1525-1538.

[31] S. Rangwala, D. Dornfeld, Integration of sensors via neural networks for detection of tool wear states, American Society of Mechanical Engineers, Production Engineering Division (Publication) PED 25 (1987) 109-120.

[32] E. Chung, Y. Chiou, S. Liang, Tool wear and chatter detection in turning via time-series modeling and frequency band averaging, American Society of Mechanical Engineers, Production Engineering Division (Publication) PED 64 (1993) 351-358.
[33] X.Q. Li, Y. Wong, A.Y.C. Nee, Comprehensive identification of tool failure and chatter using a parallel multi-ART2 neural network, J. Manuf. Sci. Eng. Trans. ASME 120 (2) (1998) 433-442.

[34] G. Byrne, D. Dornfeld, I. Inasaki, G. Ketteler, W. Konig, R. Teti, Tool condition monitoring (TCM) - The status of research and industrial application, Ann. CIRP 44 (2) (1995) 541-567.

[35] X. Li, Intelligent tool condition monitoring using wavelet and fuzzy neural network, PhD thesis, Harbin Institute of Technology, Dec. 1997. 